\title{
Adaptive Crashworthiness of Front-End Structure of Motor Vehicles
}

\author{
Marian Ostrowski, Jan Holnicki-Szulc \\ Institute of Fundamental Technological Research, Polish Academy of Sciences \\ Paulius Griskevicius \\ Kaunas University of Technology
}

Copyright $\odot 2007$ SAE International

\begin{abstract}
The aim of this research is to present a new concept of adaptive crashworthiness system. Main objectives of the proposed system are to predict, control and adjust the impact characteristics to various conditions by decreasing the pre-designed crushing stiffness of the vehicle frontal deformation zone. According to the initial pre-crash parameters (velocity, mass, stiffness and overlap) the prospective system can select proper crushing force. In most basic approach each absorber has two levels of the crushing stiffness - high and low. De-stiffening feature is driven by pyrotechnic detachable connectors integrated in the frontal car structure, which can be destroyed causing disconnection of selected structural members, excluding them from the energy dissipation process. Pyrotechnical stiffness control can be activated in a very short time, sufficient to act during typical road accidents. Among other things, the presented idea can be used to solve mass compatibility problems. Results presented in this paper show that adaptive frontal structure can help in crash performance optimization, by adjusting system properties to the various conditions of impact scenario. Decrease of the front-end crash stiffness of a striking vehicle and extension of the crushing distance can decrease occupant risk through limited side intrusion and improved deceleration pulse shape, comparing to passive systems. Detailed procedures of the crash structural control are shown. Results of simulations performed in explicit finite element code and the experimental results are demonstrated. Sensing
\end{abstract} technology is not discussed.

\section{INTRODUCTION}

Accident studies show that the crash safety performance has improved over the past years [1]. The self-protection of cars has been improved due to the introduction of various impact safety regulations and car assessment programs. However, the problem of vehicles incompatibility and partner's protection still exists. As highlighted in some studies, the crash incompatibility is more severe in side crashes [1]. Present cars are designed to fulfill front impact tests, what makes their front-end average crushing force almost proportional to their mass. In vehicle-to-vehicle side crashes, the occupants injury risk of the vehicle struck is much more severe as in striking vehicle [1]. If one of striking vehicles is heavier (front-end structure is stiffer) [2] or impact velocity is bigger, the potential of risk grows rapidly.

The variety of collision configurations provides conflicting requirements for car structures designers. One of possible solutions for crash performance optimization and car safety improvement, could be use of adaptive crashworthiness system, which is capable of adjusting energy dissipation properties to various crash conditions.

Adaptive crashworthiness system of a front-end structure was used to demonstrate a similar crash performance in both, $100 \%$ and $50 \%$ overlap, frontal collisions cases [3, 4]. Additionally, in some cases, using the adaptive structure could decrease the aggressiveness of a vehicle without compromising the self-protection. Presented adaptive system could be applicable for larger vehicles to reduce their aggressiveness towards smaller ones. Stiffness aggressiveness of the larger vehicle may be reduced by softening the front-end structure using presented system, what can decrease, comparing to passive systems, the occupant risk by improving deceleration pulse shape.

\section{FEASIBILITY DEMONSTRATOR - THEORETICAL BASE}

An experimental test was performed to check real behavior of the controllable absorber concept. Rectangular absorber shape was arbitrarily selected due to its simplicity. Main energy dissipation mechanisms in the prismatic absorber with rectangular crosssection are: the work done by traveling and stationary plastic hinges 
and the material flow through the toroidal surface in the area of crosssection corners Energy dissipation capability of the prismatic absorbers is determined by analytical formulas developed with the macroelements method. Average crushing force $P_{m}$ of the square profile can be determined by the following analytical formula [5]:

$$
P_{m}=\frac{\sigma_{0} \cdot t^{2}}{4}\left(52.22 \cdot \sqrt[3]{\frac{2 b}{t}}\right)
$$

where: $\sigma_{0}-$ plastic flow stress, $b$ - length of the rectangle side, $\mathrm{t}-$ thickness.

Dissipated energy in quasi-static crushing is equal to work of the average crushing force on the crushing distance:

$$
E_{d}=\int_{0}^{\delta} F(\delta) d \delta=P_{m} \delta
$$

where: $\delta$ - length of crushing (typically for the rectangle, full crushing distance can be assumed as $80 \%$ of the initial length).

In proposed absorber an additional energy dissipating members were designed as two lateral $\mathrm{C}$-shape profiles connected to the structure by detachable pyroconnections (Figure 1). For first calculations an arbitrary assumptions were made: the additional member is negligibly disturbing the length of the folding wave, total energy dissipated in the absorber is a simple superposition of energies of the members treated separately and there is no interaction between absorbers during its crushing. Also the length of the rectangle side differs slightly and the sheet thickness is the same, so one cross-section parameter can be used for main absorber and additional members. With the same cross-section properties also the average crushing force $\mathrm{Pm}$ is the same. Thus, estimation of the maximal energy absorbing capability in a square absorber with an additional member can be proposed by the following formula:

$$
E_{d \text { total }}=P_{m} \cdot\left(d_{b}+d_{a}\right) \cdot \alpha
$$

where: $d_{b}, d_{a}-$ initial length of the base profile and the additional member, $\alpha$ - effective crushing length factor equal to 0.8 (totally compressed absorber has $\sim 20 \%$ length of uncompressed one).

\section{FEASIBILITY DEMONSTRATOR - FEATURES}

The simple experiment and FEA simulations were performed to prove the system feasibility and perceive the "real-world" weak points of the concept. In laboratory scale tests, small impact energies were desirable. In order to decrease energy absorbing capability and forces acting on the pyro-connections (Figure 1), 1 $\mathrm{mm}$ thin sheets made of lead alloy $\mathrm{Pb} 1$ were used to manufacture the absorber. Properties of used material were: yield stress $\sigma_{Y}=8 \mathrm{MPa}$, ultimate tensile strength $\sigma_{u}=21 \mathrm{MPa}$, elongation at break $\Delta=45 \%$, and Young modulus on the level of $E=14 G P a$. The power law material model was used for the finite element calculations. No strain rate hardening was used.

A small-scale impact hammer testing stand was used, with head of the hammer weighting $37 \mathrm{~kg}$. The drop height was 1 meter over the top of the absorber. Estimated velocity at the point of the first contact with the specimen was $4.47 \mathrm{~m} / \mathrm{s}$. Basing on simplified calculation methodology shown above, the total absorbed energy of the considered laboratory lead absorber was calculated as $195 \mathrm{~J}$ for the base member and $371 \mathrm{~J}$ for the assembly. Potential energy of the hammer was $370 \mathrm{~J}$ at the point of the first contact with the absorber.
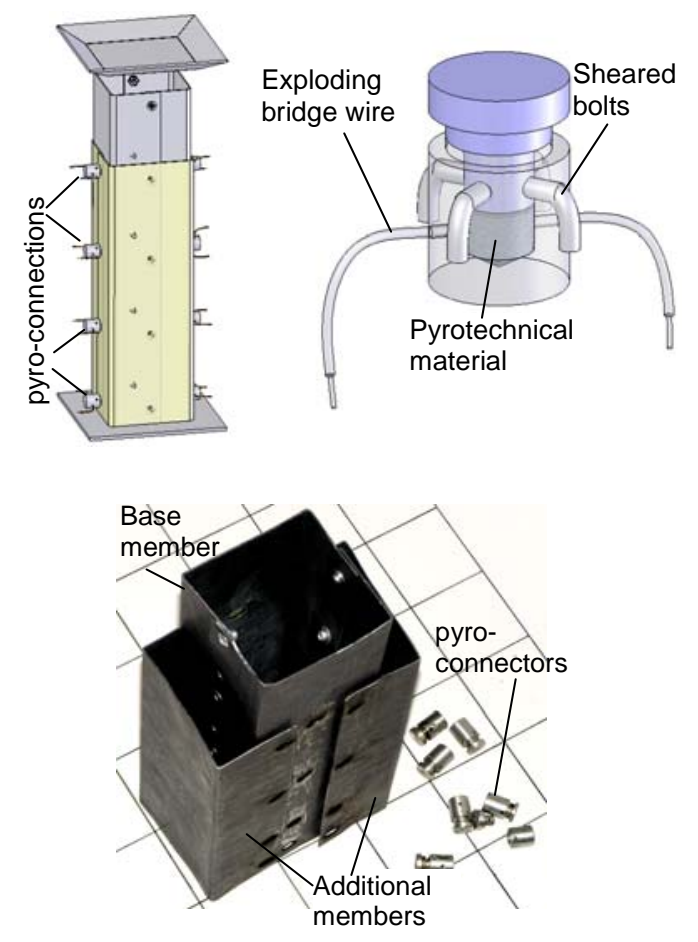

Figure 1. Absorber and its pyro-connections

Initialization of the pyrotechnical material was performed by custom designed, computer controlled, hi-voltage initiation system. Initiator made as exploding bridge wire (EBW), was initiating $0.3 \mathrm{~g}$ of black powder, closed in an explosion chamber. Rapidly increasing pressure was opening the pyro-connection, destroying sheared bolt made of soldering alloy. A capacitor-battery was charged to the $310 \mathrm{~V}$ volts and rapidly discharged through the initiator wire on the triggering signal coming from the control system. Initiator wire explodes in time shorter than $250 \mu \mathrm{s}$ after receiving the signal coming from the real-time system, driven by a specialized microcontroller. Micro-explosions were controlled by an electrical control circuit optically separated from the controller and using SCRs for fast response switching. Eight independent control channels were used. 


\section{THE EXPERIMENT}

Two cases were tested: a passive impact and active impact test. In case of the active test the pyroconnections were fired at prescribed time. FE model was simulated in explicit dynamics code LS-Dyna v971.

Passive mode test is a typical hammer crash test. The absorber was placed on the rigid base and being crashed by the moving head of the hammer. Acceleration sensor was measuring the deceleration of the impacting mass. Fast digital camera pictures were taken with the speed of 1000 frames/second (Figure 2). All pyro-connections were remained closed through the whole test time. Energy dissipation level was high.
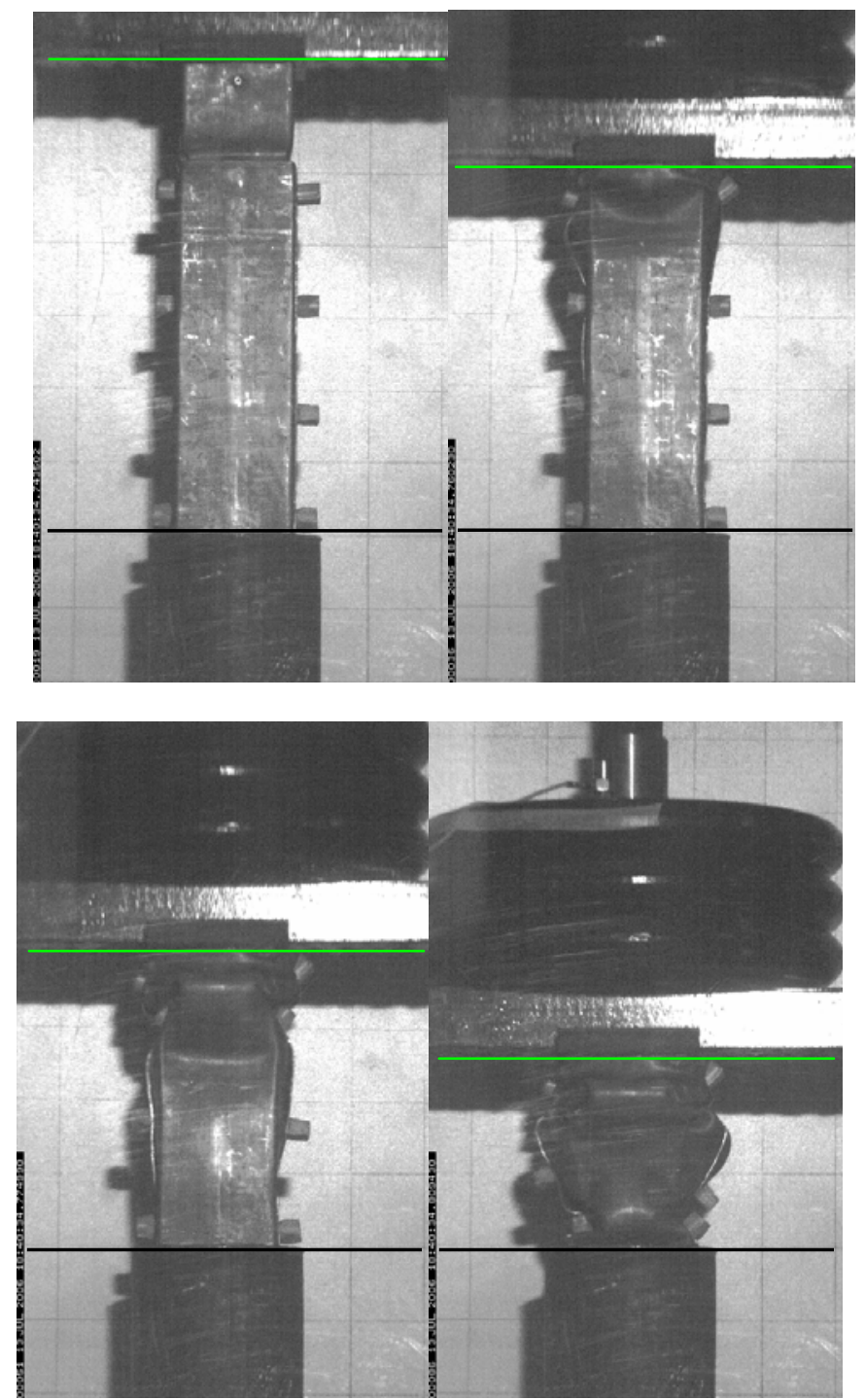

Figure 2. Results of experimental tests - absorber in passive mode

Active mode test (Figure 3) was conducted in the same conditions as the passive one. Photocell activated by the dropping head of the hammer was sending a signal to the control system. When the impulse was received by the microcontroller, the initiation system was triggered with set time offset. Statistical dispersion of explosion times was one of the main problems on early stage of the tests. Average delay time between initiation and explosion was 3-4ms. Energy dissipation level was low.
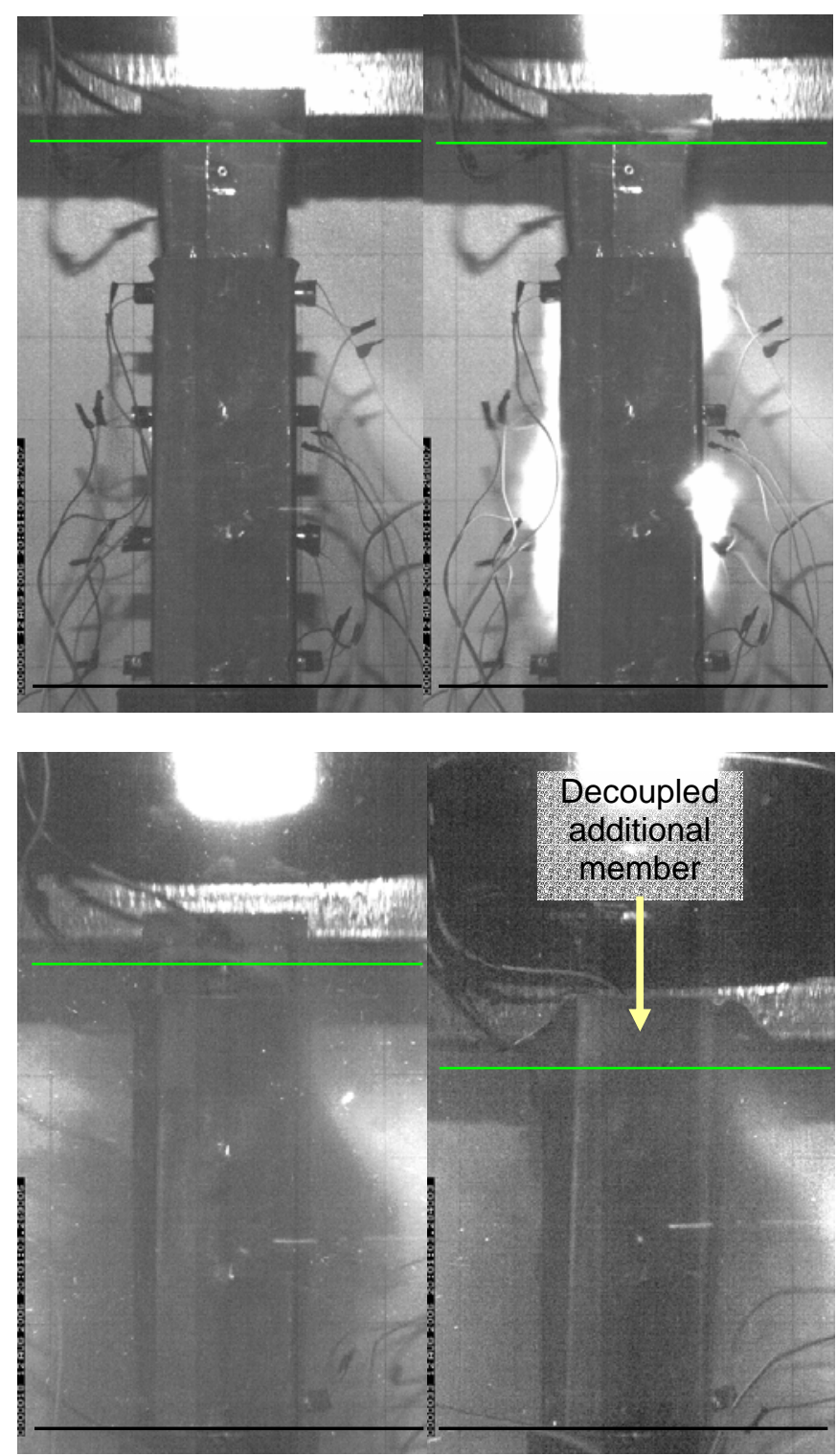

Figure 3. Results of experimental tests - absorber in active mode

Finite element simulation results for both cases are shown in the figure 4.

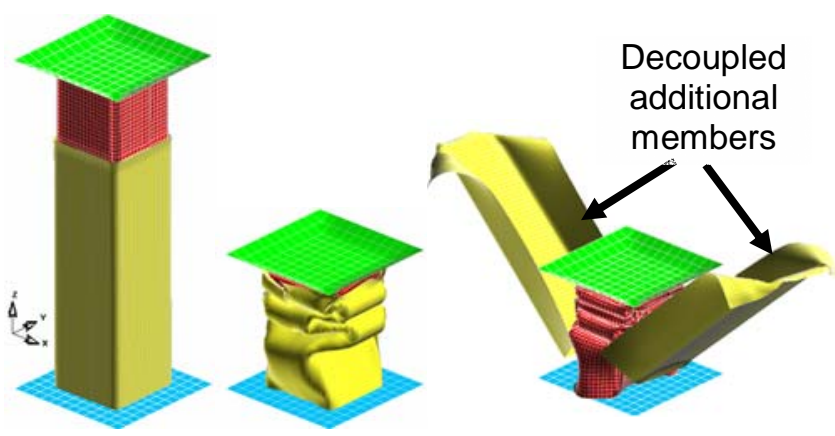

Figure 4. Deformation modes simulated in FE (form left): absorber in initial state, passive and active mode 
Plots depicting comparison of decelerations of the head of the hammer for the active and passive modes are shown in the figure 5 . On the energy plot (Figure 6) activation of the system and disconnection of the additional members is clearly visible. Time and distance of dissipation for the same amount of initial energy are longer for the low energy active mode.
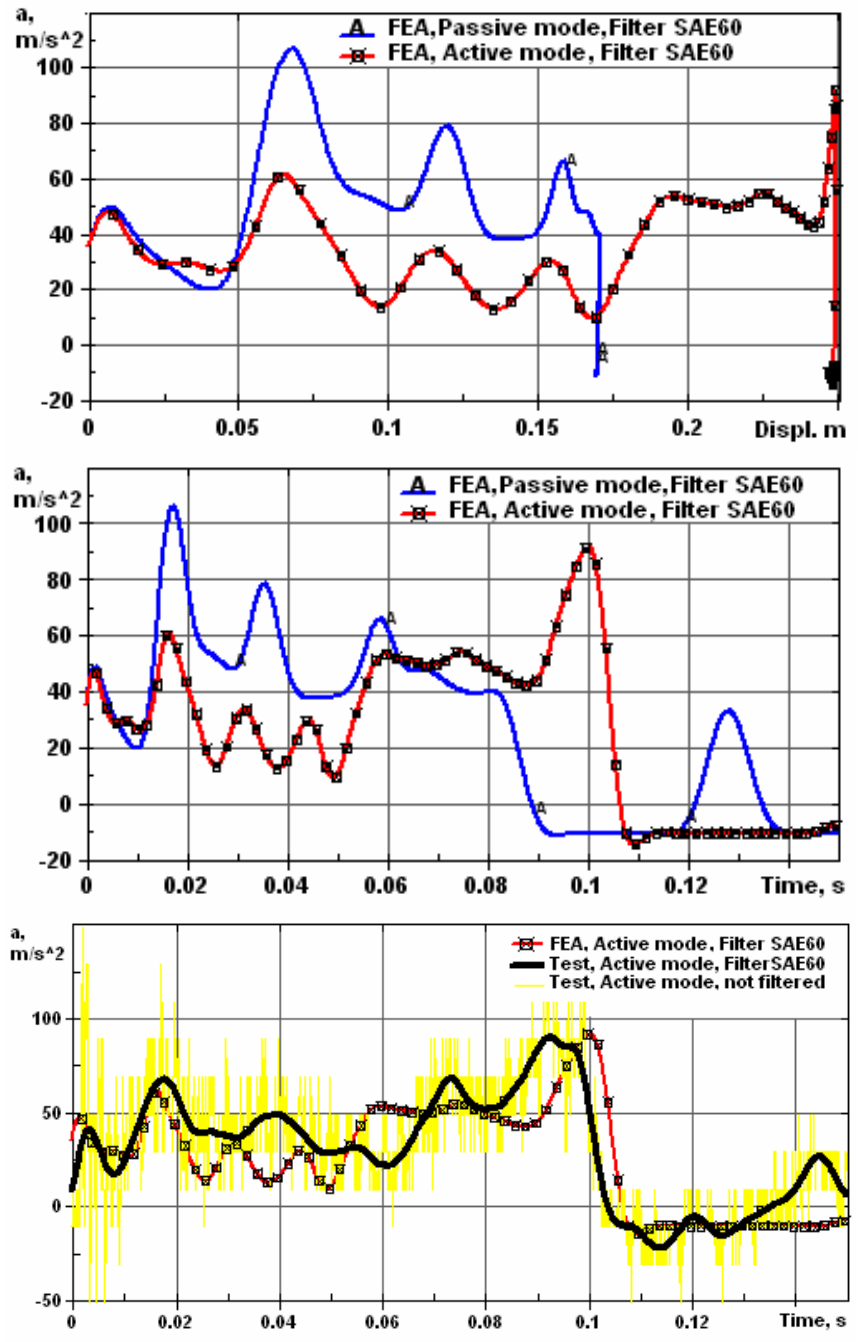

Figure 5. Decelerations of the head of the hammer in order from the top: FEA deceleration as a function of head displacement, FEA deceleration as a function of time, FEA and experimental deceleration as a function of time

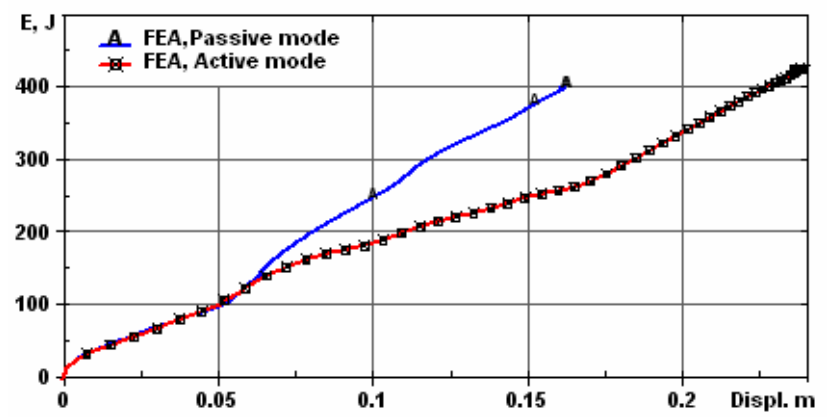

Figure 6. Dissipated energy as a function of head displacement (FEA) for passive and active mode

\section{NUMERICAL SIMULATION OF SIMPLIFIED CRASH MODEL}

The aim of the simulation was to prove that the presented adaptive structure is able to decrease the level of aggressiveness of a striking vehicle, without compromising the self-protection. Finite element simulations were performed on a simplified vehicle crash model. Adaptive energy absorber was implemented in to the FE model of vehicle front-end structure.

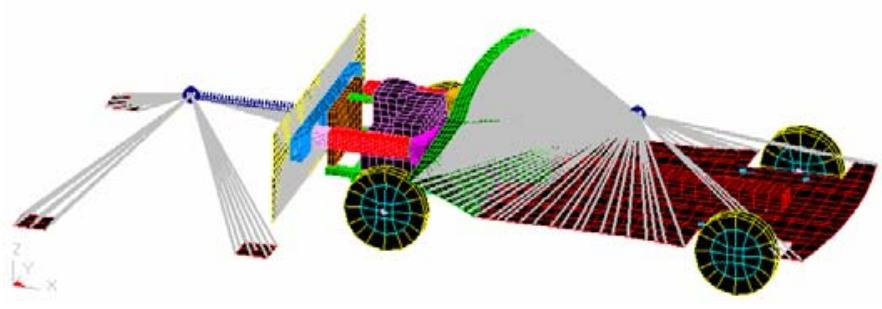

Figure 7. Simplified crash model

The FE model consists of about 20,000 nodes. Mainly quadrilateral, first order, flat Belytschko-Tsay shell elements, with Mindlin-Reissner plate theory formulation were used to model striking vehicle (Figure 7). Edge length of the shell elements in the front body structure was in range of $10 \mathrm{~mm}$ to $25 \mathrm{~mm}$. Material properties were described by *MAT_PLASTIC_KINEMATIC model with isotropic hardening and strain rate insensitivity. Additional structural members were modeled in similar way as in the feasibility demonstrator case. The FE model of vehicle front-end structure was tuned in simulations of full frontal impact to the rigid wall with velocity of impact $15 \mathrm{~m} / \mathrm{s}(54 \mathrm{~km} / \mathrm{h})$. For this case the structure pyro-connections remained closed (passive mode). Decelerations of lumped mass (Figure 8) simulating the rest of the vehicle, were used for the striking car model reliability assessment. Limit deceleration value $36 \mathrm{~g}$ was assumed to be the acceptable peak deceleration.

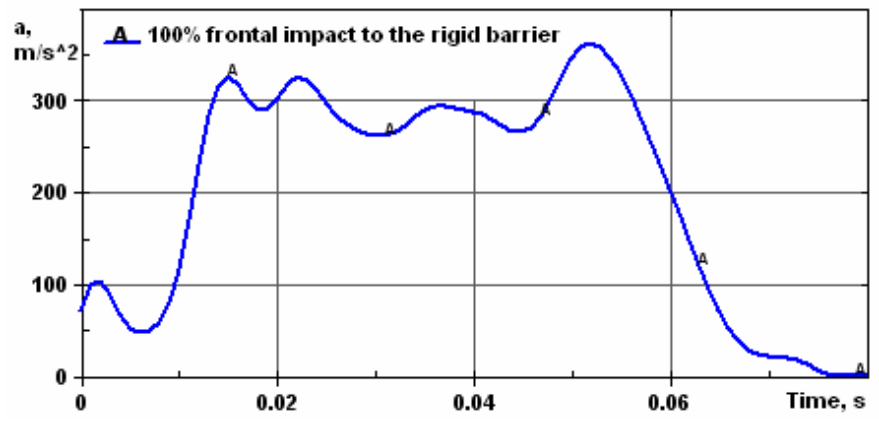

Figure 8. Deceleration pulse of full frontal impact simulations in passive mode (after SAE60 class filtering)

Side impact response of the struck vehicle was modeled in simplified way as a rigid wall connected to the point mass by a nonlinear spring. The stiffness characteristics (Figure 9) of the spring was taken from literature [6]. A lumped mass equal to $1200 \mathrm{~kg}$ was added to the centre 
of gravity. Rigid links were joining the point mass with plate shell elements simulating wheels to pavement surface contact of struck vehicle. The Coulomb friction coefficient between the plates and ground was set to the value of 0.8 .

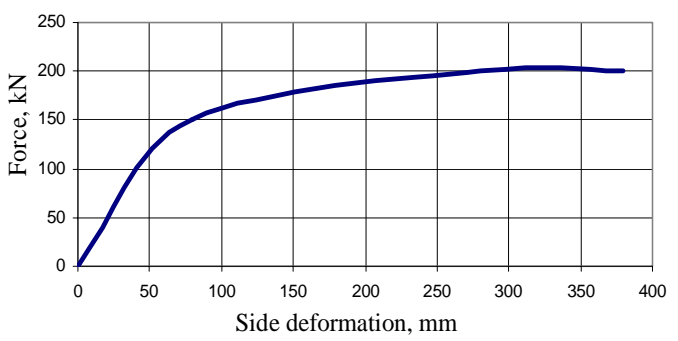

Figure 9. Side stiffness characteristics used to simulate side impact [6]

Post-impact comparison of deformations is depicted on figure 10. Adaptive system decreased deformations of struck b-pillar model and extended crushing distance on the striking vehicle front-end.

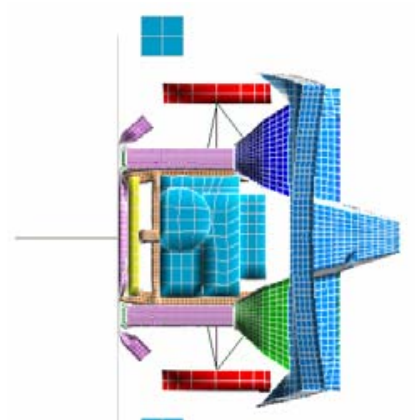

a)

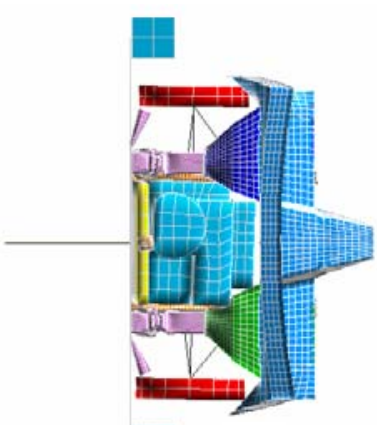

b)
Figure 10. Top view of deformed shape of front-end and b-pillar model in case of front-side impact with velocity $15 \mathrm{~m} / \mathrm{s}$ a) passive mode, b) active mode

In modern cars, front stiffness of vehicles [2] is usually much higher than side stiffness [6]. Therefore, the main safety criterion in the front-to-side crashes is intrusion of the B-pillar in to the compartment of struck vehicle. With the adaptive system, the penetration of side wall can be reduced when striking vehicle front stiffness will be decreased during impact (Figure 11).

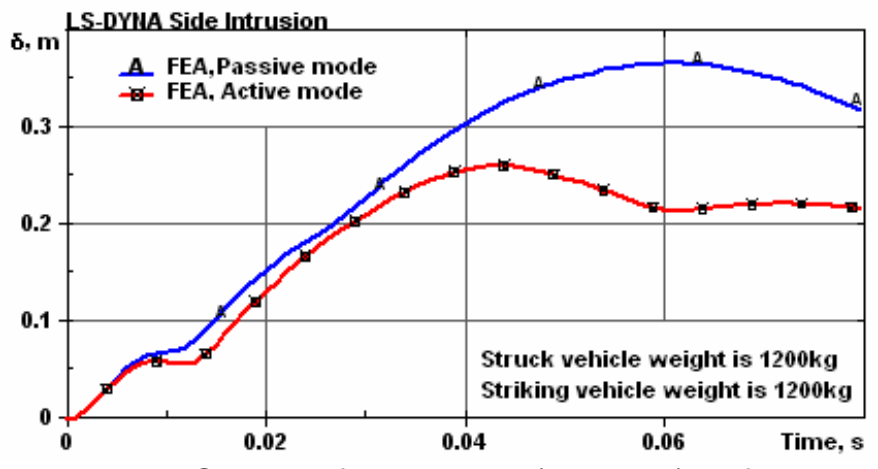

Figure 11. Side deformations (intrusion) of struck vehicles in passive and active modes

\section{CONCLUSION}

Safety improvement in front impact situations can be observed with use of adaptive crash control system. The system allows to decrease the front-end crash stiffness of striking vehicle and extend the crushing distance when it is needed. Such properties of adaptive front-end structure can decrease aggressiveness level of striking vehicle through decreased side intrusion of the struck vehicle.

\section{ACKNOWLEDGMENTS}

This paper is the final degree work done by the two authors (M. Ostrowski and P. Griskevicius) at the course of Smart Technology Expert School, held at the Institute of Fundamental Technological Research, Poland.

\section{REFERENCES}

1. Lund A.K. and Nolan J.M. Changes in vehicle designs from frontal offset and side impact crash testing. SAE, Paper No.2003-01-0902, 2003.

2. Swanson J. and Rockwell T.. Evaluation of stiffness measures from the U.S. new car assessment program. Proc. 18th International Technical Conference on the Enhanced Safety of Vehicles. Paper No. 527. Nagoya, 2003.

3. Witteman W.J. and Kriens R.F.C.. Modeling of an innovative frontal car structure: similar deceleration curves at full overlap, 40 percent offset and 30 degrees collisions. Proc. 16th International Technical Conference on the Enhanced Safety of Vehicles, Paper No. 98-S I-0-04, 1998.

4. Ostrowski M., Griskevicius P., Holnicki-Szulc J.. Feasibility study of an adaptive energy absorbing system for passenger vehicles. Proceedings of the 16th international conference on "Computer Methods in Mechanics" Czestochowa, Poland, 2005.

5. Wierzbicki T., Abramowicz W.. Mechanics of Deep Plastic Collapse of Thin-Walled Structures, 1989.

6. Digges K., Eigen A., Bahouth G. Stiffness and geometric compatibility in front-to-side crashes. SAE, Paper No.2002-01-1020, 2002.

\section{CONTACT}

Marian Ostrowski is the PhD student at the Institute of Fundamental Technological Research, Polish Academy of Sciences e-mail: mostrow@ippt.gov.pl

Jan Holnicki-Szulc is Professor at the Institute of Fundamental Technological Research, Polish Academy of Sciences and director of the SMART-TECH centre holnicki@ippt.gov.pl, http://smart.ippt.gov.pl/

Paulius Griskevicius is the head of the department of Solid Mechanics in the Kaunas university of technology (Lithuania).paulius.griskevicius@ktu.It, www.ktu.It/dkm 